\title{
Victimización directa e indirecta y sintomas de estrés postraumático en estudiantes universitarios del Noreste de México
}

\author{
Luz Adriana Orozco-Ramírez; José Luis Ybarra-Sagarduy; \\ Daniela Romero-Reyes; Oscar Armando Esparza-del-Villar
}

How to cite this article:

Orozco-Ramírez, L.A., Ybarra-Sagarduy, J.L., Romero-Reyes, D. \& Esparza-del-Villar, O.A. (2020). Direct and indirect victimization and post-traumatic stress symptoms in university students in Northeastern Mexico. Acta Colombiana de Psicología, 23(1), 301-310. doi: http://www.doi.org/10.14718/ACP.2020.23.1.14

Recibido, febrero 4/2019; Concepto de evaluación, junio 18/2019; Aceptado, agosto 18/2019

\author{
Luz Adriana Orozco-Ramírez \\ Universidad Autónoma de Tamaulipas, Tamaulipas, México \\ ORCID: https://orcid.org/0000-0003-1073-5855 \\ José Luis Ybarra-Sagarduy \\ Universidad Autónoma de Tamaulipas, Tamaulipas, México \\ ORCID: https://orcid.org/0000-0001-6405-169X \\ Daniela Romero-Reyes \\ Universidad Autónoma de Tamaulipas, Tamaulipas, México \\ ORCID: https://orcid.org/0000-0003-3239-6946 \\ Oscar Armando Esparza-del-Villar \\ Universidad Autónoma de Ciudad Juárez, Ciudad Juárez, México \\ ORCID: https://orcid.org/0000-0002-7313-3766
}

\begin{abstract}
Resumen
En México, sobre todo en los estados del noreste del país, en los últimos años la violencia se ha exacerbado y, a su vez, ha incrementado sus implicaciones en la salud mental de los jóvenes, principalmente con el desarrollo de síntomas del trastorno por estrés postraumático (TEPT). Teniendo esto en cuenta, en el presente estudio se tuvo como objetivo identificar los tipos de victimización (directa o indirecta) que viven los jóvenes universitarios ante la violencia comunitaria, así como determinar los síntomas de estrés postraumático que presentaban, e identificar la relación entre los tipos de victimización y los síntomas del TEPT. En total, participaron 500 jóvenes universitarios de un estado del noreste del país, a quienes se les aplicó la Escala de Victimización y la Escala Breve del Desorden del Estrés Postraumático. Como resultados, los participantes reportaron un promedio de siete sucesos de victimización tanto directa como indirecta, siendo más frecuente la indirecta. Aunque se observa una relación directa e indirecta entre la victimización y la presencia de síntomas del TEPT, solo la victimización indirecta resultó ser una variable predictora de síntomas del TEPT. Se propone que, junto a las políticas de prevención y reducción del delito, deben crearse políticas de reducción del miedo al crimen en la población.

Palabras clave: victimización, estrés postraumático, jóvenes universitarios, violencia comunitaria.
\end{abstract}

Unidad Académica de Trabajo Social y Ciencias para el Desarrollo Humano, Universidad Autónoma de Tamaulipas, Centro Universitario Adolfo López Mateos, Ciudad Victoria, Tamaulipas, México. C. P.: 87000. Tel.: (+52) 834-318-1800. jsagarduy@gmail.com Este proyecto fue financiado por la RED de estudios de "Violencia y Salud", reconocida ante el PRODEP-SEP 


\title{
Direct and indirect victimization and post-traumatic stress symptoms in university students in Northeastern Mexico
}

\begin{abstract}
In recent years, violence has been exacerbated in several northeastern states of México, with implications for the mental health of young people, as is the development of symptoms of post-traumatic stress disorder (PTSD). The present study aims to identify the types of victimization (direct or indirect) experienced by university students, determine the symptoms of post-traumatic stress they present and see the relationship between the types of victimization and PTSD symptoms. The Victimization Scale and the Short Scale of Post-Traumatic Stress Disorder were applied to 500 young people of Tamaulipas. The university students report on average 7 direct and indirect victimization events, the indirect being more frequent. Although there is a relationship between direct and indirect victimization, with the presence of PTSD symptoms, only indirect victimization is a predictive variable of PTSD symptoms in university students. It is proposed that, together with policies of crime prevention and reduction, policies of the reduction of fear of crimes should be created.

Key words: victimization, posttraumatic stress, university students, community violence.
\end{abstract}

\section{Introducción}

México atraviesa una grave crisis de violencia y de seguridad desde hace varios años, que se extiende a varios estados del país, principalmente en los estados fronterizos con Estados Unidos (Baja California, Sonora, Chihuahua, Coahuila, Tamaulipas), donde se producen miles de asesinatos, secuestros y extorsiones cada año. La Encuesta Nacional de Victimización y Percepción sobre seguridad pública realizada por el Instituto Nacional de Estadística, Geografía e Informática de México (INEGI, 2018) reportó un total de 25.4 millones de habitantes víctimas de $18 \mathrm{o}$ más de delitos en el 2017, lo cual representa una tasa de 29746 víctimas por cada cien mil habitantes durante ese año; además de que el costo total a consecuencia de la inseguridad y el delito en hogares representó un monto de 299.6 mil millones de pesos, lo que constituye el $1.65 \%$ del Producto Interno Bruto (PIB) de México.

La Organización Mundial de la Salud, en su informe World Report on Violence and Health: Summary publicado en español por la Organización Panamericana de la Salud (OPS, 2002), define la violencia como:

El uso intencional de la fuerza física o el poder, como amenaza o acción efectiva, contra sí mismo, contra otra persona o en contra de un grupo o comunidad, ya sea que produzca o tenga una alta probabilidad de producir una lesión (daño), la muerte, daño psicológico, mal desarrollo o la privación de la(s) persona(s) agredida(s) (p. 4).

A partir de esta definición se establece una tipología de la violencia donde la violencia comunitaria sería un tipo de violencia interpersonal, esto es, la violencia impuesta por otro individuo o un número pequeño de individuos que se produce entre personas que no guardan parentesco y que pueden conocerse o no, y que sucede por lo general fuera del hogar. Esta clasificación de violencia se debe diferenciar de otros tipos de violencia interpersonal, como lo son la violencia familiar o de pareja - que se producen sobre todo entre los miembros de la familia o de la pareja, y que, por lo general, aunque no siempre, sucede en el hogar-, y la violencia colectiva - que se refiere a la violencia infligida por grupos grandes, como grupos políticos organizados, grupos de milicia, organizaciones terroristas, etc.-(OPS, 2002).

Con respecto a la violencia comunitaria, Echeburúa (2004) señala dos tipos de posibles víctimas o afectados: (a) las víctimas directas o afectados primarios, es decir, las personas afectadas directamente por una agresión o evento traumático; y (b) las víctimas indirectas o secundarias, que son las personas que sufren algún trauma debido, por una parte, a la condición física o sociocultural que viven después de la violencia, o, por otra, por haber sido testigos indirectos, pero no haber sido personalmente afectados - dentro de esta segunda categoría se incluyen los miembros de la familia y personas cercanas a aquellos que han sido afectados de manera directa-

De manera especial, la exposición a la violencia comunitaria en los jóvenes se ha convertido en un problema de salud pública significativo en México dadas las consecuencias negativas en los diversos aspectos del desarrollo del joven. Al respecto, entre los correlatos conductuales, emocionales y académicos de la exposición a la violencia que se han estudiado en la literatura se encuentran la ansiedad, la depresión, la conducta disruptiva y violenta, el consumo de drogas, el absentismo escolar y el fracaso académico (Elsaesser, 2018; Elsaesser, Gorman-Smith, Henry \& Schoeny, M., 2017; Fowler, Tompsett, Braciszewski, Jacques-Tiura \& Baltes, 
2009; Javdani, Abdul-Adil, Suarez, Nichols \& Farmer, 2014; Wright, Fagan \& Pinchevsky, 2013; Zimmerman \& Posick, 2016); y, de manera más específica, Fowler et al. (2009) encontraron en un meta-análisis, que comprendía 114 estudios, que la exposición a la violencia comunitaria estaba muy fuertemente ligada a la aparición del trastorno de estrés postraumático (TEPT).

Ahora bien, según la American Psychiatric Association (APA, 2014), en su Manual Diagnóstico y Estadístico de los Trastornos Mentales (DSM-V), el TEPT se diagnostica cuando una persona ha desarrollado síntomas específicos tras la exposición a uno o más eventos traumáticos, y cuando la reacción emocional experimentada ante el evento traumático implica una respuesta intensa de miedo, impotencia y horror.

Respecto a este diagnóstico, Caballero y Ramos (2004) realizaron una revisión de los estudios desarrollados por el Instituto Nacional de Psiquiatría de México sobre la relación entre la violencia y el TEPT desde una metodología cualitativa, donde se encontró que en varios de ellos se utilizó la entrevista en profundidad para tratar de comprender el impacto psicológico en las víctimas de delitos - robo a casa, asalto en vía pública y violación-, así como su relación con el TEPT (Caballero, Ramos \& Saltijeral, 2000; Ramos, Caballero, Saltijeral, 1995; Ramos, Saltijeral, Caballero, 1995; Ramos, Esteban, Saltijeral, Caballero, 1997). Adicional a esto, los autores pretendían conocer las reacciones y el impacto psicológico que presentan las víctimas de dichos delitos tanto en el primer mes como a los seis meses de ocurrido el hecho y su relación con el TEPT, así como el impacto subjetivo del episodio de violencia.

Como resultados, prácticamente todas las víctimas de asalto en vía pública expresaron un miedo a sufrir daños físicos o a morir, mientras que los robos a las casas fueron experimentados sobre todo como una violación o invasión a la intimidad que se vive muy intensamente. De igual forma, y con una alta frecuencia, las víctimas de estos delitos tendieron a presentar una re-experimentación del suceso en forma de recuerdos repetitivos e incontrolables, y otra reacción frecuente fue el malestar psicológico, según el cual los entrevistados manifestaron, principalmente, miedo a que se repita el incidente. También fue bastante común encontrar que los sujetos se esforzaban por no tener pensamientos y sentimientos asociados con el robo, es decir, conductas evitativas (Caballero \& Ramos, 2004).

Por otra parte, también se han realizado estudios desde una metodología cuantitativa, como el trabajo de Baker et al. (2005), en el que se entrevistó a una muestra de 2509 adultos de cuatro ciudades de México (Oaxaca, Guadalajara, Hermosillo y Mérida), utilizando la Entrevista Diagnóstica Internacional de Salud Mental (Composite International Diagnostic Interview, CIDI). En este estudio, el $34 \%$ de la muestra informó haber sufrido por lo menos una vez a lo largo de su vida un evento relacionado con uno de cuatro tipos de violencia - el asalto sexual, el abuso sexual, el asalto físico o la amenaza con un arma- $-\mathrm{y}$, para aquellos que reportaron al menos un evento violento a lo largo de su vida $(\mathrm{N}=855)$, el $11.5 \%$ cumplió los criterios para el TEPT relacionado con la violencia; incluso se asociaron con mayores probabilidades de TEPT la violencia de tipo sexual sufrida con la pareja íntima, siendo mayor en las mujeres que en hombres.

Por otra parte, Orozco, Borges, Benjet, Medina-Mora y López-Carrillo (2008) aplicaron el mismo instrumento que Baker et al. (2005) con adolescentes de la Zona Metropolitana de la Ciudad de México, y sus resultados arrojaron que el $68.9 \%$ reportó por lo menos un evento traumático alguna vez en su vida, con diferencias por sexo; además de que la prevalencia del TEPT fue del $1.8 \%$ ( $2.4 \%$ en mujeres y $1.2 \%$ en hombres), y el abuso sexual se asoció al desarrollo del TEPT, independientemente del sexo, educación o edad; y que el $28.2 \%$ de los jóvenes mencionó haber vivido dos o tres eventos traumáticos, mientras que el $13 \%$ reportó cuatro o más.

Asimismo, Leal, Vázquez y Cantú (2013) realizaron un estudio que trató de determinar la prevalencia del TEPT en unidades de medicina familiar (UMF) de seis ciudades fronterizas del norte del estado de Tamaulipas, México, y sus resultados mostraron que el TEPT fue más frecuente en mujeres y que los factores relacionados más comunes fueron el robo y la violación o abuso sexual. Más recientemente, Rizo, Guevara, Hernández y Sánchez-Sosa (2018) trataron de examinar la prevalencia de síntomas de TEPT junto con otras psicopatologías como la depresión y la ansiedad en adolescentes víctimas de maltrato en México, y observaron que las adolescentes que experimentaron alguna forma de abuso presentaban una alta susceptibilidad a desarrollar psicopatología. En definitiva, en México son escasos los estudios que exploran de manera directa la violencia comunitaria a la que han sido expuestos los jóvenes o que analizan su relación con la sintomatología del TEPT, y las investigaciones existentes no diferencian de manera explícita entre violencia primaria-directa o la secundaria-indirecta ni cómo se relaciona cada una con los síntomas del TEPT.

Finalmente, en el presente artículo se muestran resultados que son una continuación de los obtenidos en la investigación de Almanza, Gómez y Gurrola (2017) -quienes tenían como objetivo analizar la relación entre la victimización directa e indirecta, la resiliencia y la sintomatología psicológica-; en dicha investigación, los autores reportaron un papel moderador de la resiliencia en el impacto de la victimización directa e indirecta en la 
sintomatología psiquiátrica en las dimensiones de somatización, obsesión-compulsión, sensibilidad interpersonal, depresión, ansiedad, miedo, hostilidad, ansiedad fóbica, ideación paranoide, psicoticismo; sin embargo, en dicho estudio no se tuvieron en cuenta los síntomas del TEPT, por lo que en el presente trabajo se utilizó la misma muestra del estudio anteriormente mencionado y se tuvo como objetivo identificar los tipos de victimización (directa o indirecta) derivados del contexto local generados por la violencia comunitaria en jóvenes universitarios, así como determinar sus síntomas de estrés postraumático, identificar la relación entre los tipos de victimización y los síntomas del TEPT, y analizar si la edad, el género o el tipo de victimización predicen o no los síntomas del TEPT.

\section{Método}

\section{Tipo de investigación}

Se realizó un estudio empírico con metodología cuantitativa. Concretamente, se trata de un estudio ex post facto de tipo retrospectivo, con un solo grupo y medidas múltiples (Montero \& León, 2007).

\section{Participantes}

En el estudio participaron alumnos de una universidad pública de Ciudad Victoria, capital del estado de Tamaulipas, situada en el noreste de México. Se realizó un muestreo no probabilístico por cuotas, estableciendo una cuota de 100 participantes de las facultades de Derecho, Ingeniería, Enfermería, Administración y Ciencias de la Educación, a fin de contar con la participación de estudiantes de diversas carreras. De los 509 participantes que respondieron, se suprimieron nueve $(1.8 \%)$ que no contestaron la batería completa, quedando un total de 500 participantes.

Como características principales se observa que las edades de los participantes se encontraban entre los $17 \mathrm{y}$ los 26 años $(X=19.9$; DT = 1.90); que el $51 \%(n=257)$ eran mujeres y el $49 \%(n=243)$, varones; que el $89.2 \%$ eran solteros, principalmente de los semestres primero a sexto $(90.3 \%)$; que el total de los participantes contaba con una media de rendimiento académico de 8.99; que el $82 \%$ estudiaba en el turno vespertino; que solo el $24.3 \%$ trabajaba; que el $50.5 \%$ realizaba una actividad deportiva; $y$ que solo el $16 \%$ hacía alguna actividad artística o cultural.

\section{Instrumentos}

Los datos sociodemográficos de la muestra se recabaron a través de una breve cedula en la que se solicitó información sobre edad, género, carrera, semestre, promedio académico, actividades extraescolares, estado civil y si trabajaban en la actualidad. Además, se aplicó la Escala de Victimización y la Escala breve del Desorden del Estrés Postraumático.

Escala de Victimización (Ruíz, 2007a). Esta escala está compuesta por diferentes situaciones violentas catalogadas en dos niveles: el "nivel primario/directo (a mí personalmente)", con 15 ítems, y el "nivel secundario/indirecto (a tu pareja, un familiar o conocido cercano)", con 17 ítems. Cada una de las situaciones violentas expuestas se tiene dos opciones de respuesta ("Sín" o "No"), y entre ellas se encuentran el robo, la extorsión, la agresión sexual y el secuestro, entre otras.

En particular, esta escala se trata de una lista de verificación que se ha utilizado en otros estudios con poblaciones de adultos jóvenes, como en Ruiz (2007b), Ruiz y Turcios (2009) o Muratori y Zubieta (2013), pero sin informarse de resultados psicométricos. En el presente estudio se realizó una validación de contenido de la escala mediante el juicio de expertos y, con base en los resultados, se agregaron tres ítems para considerar las formas específicas en que se manifiesta la violencia comunitaria en el contexto local: "balaceado", "detenido en falsos retenes" y "agresiones por parte del ejército". Al sumarse cada nivel, se puede obtener una puntuación total de 0 a 18 en nivel primario y de 0 a 20 puntos en nivel secundario. Mediante la aplicación del coeficiente de Kuder-Richardson (KR-20) para escalas con ítems de respuesta dicotómica, la escala presentó niveles aceptables de consistencia interna tanto para la victimización directa $(\alpha=.787)$ como para la indirecta $(\alpha=.888)$.

Escala breve del Desorden del Estrés Postraumático (Breslau, Peterson, Kessler \& Schultz, 1999).

Esta escala está compuesta por siete ítems con dos opciones de respuesta ("Sí" o "No"), donde el participante debe indicar los síntomas que ha presentado después de haber vivido o presenciado algún acto de violencia. La escala posee un $78 \%$ de sensibilidad, un $97 \%$ de especificidad, un $75 \%$ de valor predictivo positivo, y un $98 \%$ de valor predictivo negativo (Bohnert \& Breslau, 2011). Adicional a esto, el instrumento cuenta con un punto de corte de cuatro, para un cribaje de síntomas de TEPT, y en el presente estudio mostró una consistencia interna adecuada, de .79.

\section{Consideraciones éticas}

El proyecto fue aprobado por el Programa para el Desarrollo Profesional Docente (PRODEP) de la Secretaria de Educación Superior de México, y se llevó a cabo según las normas contempladas en el Código Ético del Psicólogo (Sociedad Mexicana de Psicología, 2010). 


\section{Procedimiento}

Inicialmente, se elaboró un oficio con los datos de las facultades participantes con el fin de obtener autorización para la aplicación de los instrumentos. Después, al otorgarse la aceptación por parte de los directivos de las facultades, se llevó a cabo la aplicación de los instrumentos en cada salón autorizado, empezando con la lectura de las instrucciones por parte del investigador, donde, además, se ofreció información sobre la utilidad de los resultados de los instrumentos y se dejó en claro la importancia de la participación de todos los estudiantes. Antes de empezar a contestar los instrumentos, cada participante debía firmar un consentimiento informado donde se les indicaba que la participación era voluntaria y que eran libres de retirarse en cualquier momento del estudio, sin que ello afectara su situación académica; y que los datos serían manejados de forma confidencial y anónima. Seguido a esto, comenzó la aplicación de instrumentos, atendiendo las dudas que se iban generando en el transcurso de la aplicación hasta que cada joven terminaba de contestar.

\section{Análisis de datos}

En un primer momento, se obtuvieron los estadísticos descriptivos de las variables de interés del estudio (victimización directa e indirecta y síntomas del TEPT) y se verificó mediante la prueba de Kolmogorov-Smirnov la distribución normal de los datos. Posteriormente, se estudió la relación entre las variables mediante la aplicación de la prueba no paramétrica de correlación Rho de Spearman y se analizó la asociación entre las posibles situaciones de violencia que llevan a la victimización directa o indirecta y a los síntomas del TEPT con la prueba chi-cuadrada y el coeficiente phi $(\phi)$. Por último, se realizó un análisis de regresión logística con el método por pasos hacia adelante condicional, con la presencia de síntomas del TEPT como variable dependiente. En un primer paso se introdujeron en el modelo como variables independientes la edad y el género; en un segundo paso se introdujeron las variables de victimización directa e indirecta; y, por último, en un tercer paso se introdujo la variable de interacción entre victimización directa e indirecta.

\section{Resultados}

Los estudiantes universitarios reportaron haber sido víctimas de una situación violenta dos veces en promedio de manera directa $(X=2.19$, DT $=2.69)$ y cinco veces siendo victimas indirectas $(\mathrm{X}=5.27, \mathrm{DT}=4.89)$, es decir, con actos violentos sufridos por un familiar o persona cercana. Asimismo, el $65.6 \%(\mathrm{n}=328)$ de la muestra reportó haber sido víctima directa de al menos un suceso de violencia comunitaria en su vida, mientras que el $81.8 \%$ $(\mathrm{n}=409)$ reportó haber sido víctima indirecta al menos una vez en la vida.

En particular, las situaciones de victimización directa más frecuentes fueron el ser perseguidos por desconocidos, en un $27 \%(\mathrm{n}=133)$; recibir llamadas obscenas, $24 \%$ $(\mathrm{n}=122)$; ser víctimas de extorsión económica, $17 \%$ $(\mathrm{n}=86)$; ser víctimas de robo en vivienda, $17 \%(\mathrm{n}=84) ; \mathrm{y}$ ser víctimas de robo de objetos en su carro, $17 \%(\mathrm{n}=84)$. Mientras que, en cuanto a la victimización indirecta, las situaciones más frecuentes fueron el robo en la vivienda de algún familiar o persona cercana, en un $46 \%(n=228)$; extorsión económica a un familiar o persona cercana, $42 \%$ $(n=208)$; robo de objetos en el carro de un familiar o persona cercana, $37 \%(n=183)$; y tentativa de robo a un familiar o persona cercana, $33 \%(\mathrm{n}=169)$.

Cabe destacar que existen situaciones que, aun no siendo las más frecuentes, se viven en el contexto de la lucha contra el crimen organizado que se experimenta en el noreste de México. En este contexto, en las situaciones de victimización directa se encuentra que un $13 \%(n=63)$ reportó muerte violenta cercana a mí, y que uno de cada diez había sido detenido en falsos retenes; mientras que en cuanto a la victimización indirecta, el $24 \%(n=118)$ había sido víctima de la desaparición de algún familiar y dos de cada diez tenían familiares cercanos que habían sido detenidos en falsos retenes.

Con respecto a los síntomas del TEPT, se encontró que el $20 \%(\mathrm{n}=98)$ de los estudiantes universitarios ya presentaba un diagnóstico positivo de síntomas del TEPT, y los síntomas que mostraron mayor porcentaje en la muestra fueron "ha disminuido mi interés por actividades que antes eran placenteras", con un $45.7 \%$, y "hago esfuerzos por evitar actividades, lugares o personas que me recuerden el hecho violento", con un $34.3 \%$ (véase Figura 1).

Ahora bien, la prueba de normalidad de KolmogorovSmirnov para las tres variables, síntomas de estrés postraumático y victimización directa e indirecta, resultó significativa $(\mathrm{p}<.000)$, por lo que se concluye que no hay una distribución normal de los datos, $\mathrm{y}$, al relacionar los tipos de victimización con los síntomas del TEPT mediante la prueba no paramétrica Rho de Spearman, se encuentran relaciones positivas significativas bajas que indican que a mayor victimización directa $(\mathrm{r}=.251 ; \mathrm{p}=.000)$ y mayor victimización indirecta $(r=.267 ; \mathrm{p}=.000)$, mayores síntomas del TEPT. 


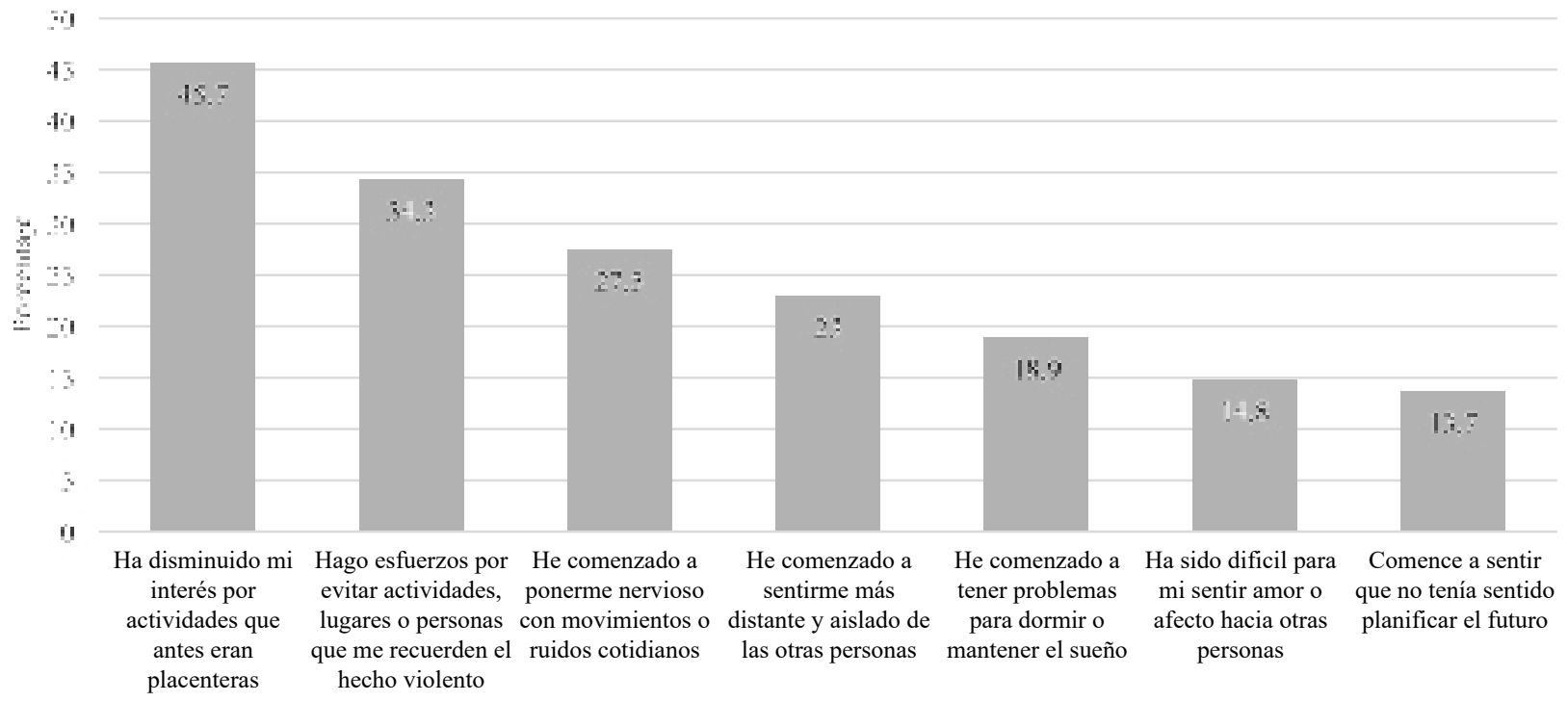

Figura 1. Síntomas del trastorno por estrés postraumático. Fuente: elaboración propia.

También, dentro de las situaciones de victimización directa asociadas a los síntomas del TEPT se encontraron: tentativa de robo $\left(\chi^{2}=6.141, \mathrm{p}=.017\right)$, vandalismo en el carro $\left(\chi^{2}=4.475, \mathrm{p}=.039\right)$, extorsión económica $\left(\chi^{2}=9.710\right.$, $\mathrm{p}=.004)$, ser perseguido por desconocidos $\left(\chi^{2}=25.678\right.$, $\mathrm{p}=.000)$, robo de objetos en el carro $\left(\chi^{2}=9.710, \mathrm{p}=.004\right)$ y ser detenido en falsos retenes $\left(\chi^{2}=5.660, \mathrm{p}=.023\right.$ ) (véase Tabla 1). Sin embargo, la prueba phi $(\phi)$ muestra que estás situaciones tienen un grado de asociación bajo, siendo el ser perseguido por desconocidos la única situación con un valor phi superior a 2 .

Asimismo, en el caso de la victimización indirecta las situaciones relacionadas con los síntomas del TEPT fueron el robo de moto o bicicleta a un familiar $\left(\chi^{2}=4.826\right.$, $\mathrm{p}=.038)$, vandalismo en el carro $\left(\chi^{2}=4.203, \mathrm{p}=.044\right)$, robo con violencia a un familiar $\left(\chi^{2}=7.065, \mathrm{p}=.011\right)$, agresión o amenazas físicas a un familiar $\left(\chi^{2}=8.124\right.$, $\mathrm{p}=.006)$, secuestro a un familiar $\left(\chi^{2}=5.377, \mathrm{p}=.023\right)$, extorsión económica $\left(\chi^{2}=5.465, \mathrm{p}=.022\right)$, ser perseguido por desconocidos $\left(\chi^{2}=17.914, \mathrm{p}=.000\right)$, llamadas obscenas a un familiar $\left(\chi^{2}=7.866, p=.007\right)$, muerte violenta de una persona cercana $\left(\chi^{2}=5.155, \mathrm{p}=.033\right)$, robo de objetos en el carro $\left(\chi^{2}=12.260, \mathrm{p}=.001\right)$, tener un familiar balaceado $\left(\chi^{2}=7.582, \mathrm{p}=.009\right)$, ser detenido en falsos retenes $\left(\chi^{2}=8.522, p=.006\right)$ y agresiones por parte del ejército $\left(\chi^{2}=8.052, \mathrm{p}=.009\right)$ (véase Tabla 1). Acá, las pruebas phi $(\phi)$ muestran que el grado de asociación de todas estas situaciones es bajo, ninguno supera el valor 2 .
Finalmente, con respecto al análisis de regresión logística realizado con el método por pasos hacia adelante condicional para examinar las variables predictoras de síntomas de estrés postraumático (véase Tabla 2), la única variable que resultó predictora fue la victimización indirecta $($ Wald $=6.027, \operatorname{Exp}[\mathrm{B}]=1.077, \mathrm{IC}$ de $95 \%$ [1.037, $1.131], \mathrm{p}<.05)$. Respecto al ajuste de este modelo, el cambio significativo en la desvianza muestra un ajuste global significativo $\left(\mathrm{X}^{2}=12.935, \mathrm{p}<.0001\right)$, confirmado con el Test de Hosmer-Lemeshow $\left(\mathrm{X}^{2}=4.873, \mathrm{p}=.771\right)$, y permite hacer una estimación correcta del $80.3 \%$ de los casos. Por otro lado, el coeficiente R2 de Nagelkerke indica que el modelo de regresión logística explica el $4.2 \%$ de la varianza de la variable dependiente.

\section{Discusión}

El objetivo del presente estudio consistió en identificar la relación entre la victimización ante la violencia comunitaria y los síntomas del TEPT, estableciendo una diferencia entre la violencia primaria o directa y la secundaria o indirecta. Como resultado, se encontró que el $65.6 \%$ de la muestra había sido víctima directa de al menos un suceso de violencia comunitaria en su vida, hallazgo similar a estudios previos, como el de Medina-Mora et al. (2005) y el de Orozco et al. (2008). Sin embargo, y a diferencia de estos dos, en el presente trabajo se estudió adicionalmente la violencia secundaria, con lo que se encontró que el 81.8 \% informó 
Tabla 1.

Asociación entre las posibles situaciones de victimización directa e indirecta con los síntomas del estrés postraumático

\begin{tabular}{|c|c|c|c|c|}
\hline & \multicolumn{2}{|c|}{$\begin{array}{l}\text { Victimización directa/ } \\
\text { Síntomas de estrés postraumático }\end{array}$} & \multicolumn{2}{|c|}{$\begin{array}{l}\text { Victimización indirecta/ } \\
\text { Síntomas de estrés postraumático }\end{array}$} \\
\hline & $\chi^{2}$ & $\phi$ & $\chi^{2}$ & $\phi$ \\
\hline Robo en vivienda & 1.025 & .046 & 0.929 & .043 \\
\hline Tentativa de robo en vivienda & $6.141^{*}$ & $.111^{*}$ & 1.869 & .061 \\
\hline Robo de carro & 1.024 & .045 & 3.800 & .088 \\
\hline Robo de moto o bicicleta & 0.076 & .012 & $4.826 * *$ & $.099 *$ \\
\hline Vandalismo en el carro & $4.475^{*}$ & $.095^{*}$ & $4.203^{*}$ & $.092 *$ \\
\hline Robos con violencia e intimidación & 2.776 & .075 & $7.065^{*}$ & $.119 * *$ \\
\hline Robos sin violencia & 0.062 & .011 & 0.013 & .005 \\
\hline Agresión sexual & 0.038 & .009 & 2.966 & .077 \\
\hline Agresión física o amenazas & 0.312 & .025 & $8.124 * *$ & $.129 * *$ \\
\hline Secuestro & 0.034 & .008 & $5.377^{*}$ & $.104 *$ \\
\hline Extorsión económica & $9.710 * *$ & $.140 * *$ & $5.465^{*}$ & $.105^{*}$ \\
\hline Ser perseguido por desconocidos & $25.678 * *$ & $.228 * * *$ & $17.914 * *$ & $.190 * * *$ \\
\hline Llamadas obscenas de desconocidos & 2.474 & .071 & $7.866^{* *}$ & $.126^{* *}$ \\
\hline Muerte violenta de una persona cercana & 3.499 & .084 & $5.155^{*}$ & $.102 *$ \\
\hline Robo de objetos del carro & $9.710 * *$ & $.140 * *$ & $12.260 * *$ & $.157 * * *$ \\
\hline Desaparición & - & - & 1.797 & .060 \\
\hline Homicidio & - & - & 1.084 & .047 \\
\hline Balaceado & 0.407 & .029 & $7.852 * *$ & $.124 * *$ \\
\hline Detención en falsos retenes & $5.660 *$ & $.107^{*}$ & $8.522 * *$ & $.131 * *$ \\
\hline Agresión por el ejercito & 1.626 & .057 & $8.052 * *$ & $.128 * *$ \\
\hline
\end{tabular}

Nota. $* \mathrm{p}<.05, * * \mathrm{p}<.01 * * * \mathrm{p}<.001$. Fuente: elaboración propia.

Tabla 2.

Análisis de regresión logística de la presencia de sintomas del trastorno por estrés postraumático

\begin{tabular}{lcccccc}
\hline & B & E. T. & Wald & gl & Sig. & Exp(B) \\
\hline Sexo & .086 & .229 & 0.139 & 1 & .709 & 1.089 \\
Edad & .050 & .062 & 0.653 & 1 & .419 & 1.051 \\
Victimización directa & .073 & .047 & 2.449 & 1 & .118 & 1.076 \\
Victimización indirecta & .074 & .030 & 6.027 & 1 & .014 & 1.077 \\
Interacción victimización directa-indirecta & .001 & .004 & 0.087 & 1 & .768 & 1.001 \\
\hline
\end{tabular}

Nota. Fuente: elaboración propia.

haber sido víctima indirecta al menos una vez en la vida. En general, los jóvenes universitarios habían experimentado aproximadamente siete sucesos de victimización, fuera directa o indirecta, tal como lo reportan otros estudios realizados en muestras latinoamericanas (Rojas-Flores, Currier, Lin, Kulzer \& Foy, 2013).

Dentro de los sucesos de victimización directa, los tres más frecuentes fueron ser perseguidos por desconocidos, recibir llamadas obscenas y la extorsión económica; y los más frecuentes en la victimización indirecta fueron el robo en vivienda, la extorsión económica y el robo de carro a algún familiar. De igual forma, se encontró que uno de cada diez participantes había sido detenido por falsos retenes directamente, que dos de cada diez tenían familiares cercanos habían sido detenidos en falsos retenes, y que el $24 \%$ había sido víctima de la desaparición de algún familiar. Con estos datos, se observa que la victimización por dichos sucesos se encuentra enmarcada por el contexto social que se vive en el noreste de México, debido a que en este hay una elevada exposición a experiencias violentas derivadas de la delincuencia relacionada con el crimen organizado. Al respecto, la encuesta "Los Miedos de México" realizada por Buendía y Laredo (2010) indica que el $38 \%$ de los mexicanos menciona que la experiencia violenta que más teme es el secuestro o que alguien de su familia sea víctima de este delito, incluso es más temido que un terremoto; por lo tanto, hay una mayor vulnerabilidad a ser víctimas de la extorsión telefónica y al temor de ser secuestrados. 
En los últimos años, la violencia y la inseguridad pública han estado afectando la convivencia y el desarrollo armónico de la región fronteriza del norte de México que comprende los estados de Baja California, Chihuahua, Coahuila, Nuevo León, Sonora y Tamaulipas. Expresiones tales como impunidad, delincuencia organizada, narcotráfico, secuestro, homicidios, "ejecuciones", "ajustes de cuentas", robos, conductas delictivas juveniles, programas de seguridad fallidos, desconfianza y corrupción en los cuerpos policiacos, y discrecionalidad en la aplicación de la ley, forman parte de la experiencia diaria de una ciudadanía lastimada, dolida, temerosa y desconfiada de las instituciones encargadas de la procuración de justicia en los tres niveles de gobierno, lo cual representa consecuencias negativas en su calidad de vida y en su salud mental (Monárrez \& García, 2008).

En general, los resultados del presente estudio indican que, en los jóvenes universitarios, a mayor victimización, sea directa o indirecta, mayores síntomas de estrés postraumático, aunque las correlaciones obtenidas fueron bajas. Esto concuerda con diferentes estudios que han señalado cómo la naturaleza, la gravedad y los efectos acumulativos de la exposición a eventos violentos son factores de riesgo para la aparición del TEPT (Brown, Hill \& Lambert, 2005; Fowler et al., 2009; Scarpa, Haden \& Hurley, 2006); o, como indica la Organización Mundial de la Salud (2014), existen diversas consecuencias en la salud mental de los habitantes que viven en contextos violentos, y dentro de estas se encuentra el TEPT. De igual manera, la exposición indirecta a eventos violentos, como presenciar, escuchar sobre actos violentos o conocer personas cercanas involucradas en actos violentos son un factor clave para el desarrollo de síntomas del TEPT (Walling, Eriksson, Putman \& Foy, 2011). Estos resultados concuerdan con la teoría de la victimización propuesta por Vilalta (2012), en la que se destaca la relación entre la victimización directa o indirecta y los daños psicológicos que originan el miedo al delito y a la inseguridad.

Otro resultado a destacar es que la única situación de victimización que muestra un mayor grado de asociación con los síntomas del TEPT es ser perseguido por desconocidos, en este caso de tipo directo, lo cual se relacionaría con investigaciones que muestran que el secuestro es el tipo de violencia que más temen los mexicanos (Buendía \& Laredo, 2010). En este estudio, sin embargo, no se encontró asociación entre sufrir agresión sexual y el desarrollo del TEPT, cuando investigaciones previas indicaron que este tipo de agresiones está más relacionado con el surgimiento de los síntomas del TEPT (Baker et al., 2005; Leal et al., 2013; Orozco et al., 2008). Una posible razón es que los participantes de este estudio presentan bajos porcentajes con respecto a haber sufrido este tipo de agresiones $(4.4 \%$ a nivel directo y $10.4 \%$ a nivel indirecto), por lo que no se pueden tener resultados concluyentes en este aspecto.

Por otra parte, se destaca que en los resultados de este estudio la variable que predice en mayor medida el desarrollo de síntomas de estrés postraumático es la victimización indirecta, y no la victimización directa, la edad ni el género. Al respecto, como menciona Ruíz (2007b), el miedo a ser víctima de una agresión está relacionado con la probabilidad que la persona estima de ser víctima de un delito. Entonces, entre más cercana se encuentra la experiencia, por ejemplo, que le haya pasado a algún familiar cercano - 0 ser testigo- incrementa el miedo de ser víctima directa. Así, debido a que los jóvenes universitarios en el noreste del país están expuestos tanto directa como indirectamente a sucesos violentos generados por el contexto social del crimen organizado, presentan también una mayor vulnerabilidad de desarrollar síntomas de estrés postraumático, principalmente los que han vivido mayores experiencias de victimización indirecta, como el secuestro, ser baleado o saber de agresiones por parte del ejercito hacia algún familiar o persona cercana.

Finalmente, este estudio afirma que la variable que predice la aparición de síntomas del TEPT es la victimización indirecta, $\mathrm{y}$, al respecto, el miedo a ser víctima de un acto de violencia, pese a su importancia en la calidad de vida, es una de las áreas menos atendidas a pesar de que impacta de forma negativa las rutinas o hábitos personales, desincentivando o limitando la realización de actividades que se deberían poder hacer libremente en la cotidianidad; este miedo causa un detrimento en el estado anímico y el desarrollo de síntomas del TEPT. Por lo tanto, de esto se concluye que las políticas de reducción de miedo al crimen deberían considerarse tan importantes como las políticas de prevención y reducción del crimen.

Cabe mencionar algunas limitaciones del estudio, como el tipo de muestra, ya que hicieron parte del estudio estudiantes universitarios, y es probable que esta población sea más privilegiada que la persona promedio en el noreste del país; de la misma forma, este estudio no consideró la variable de nivel socioeconómico, la cual resulta de gran importancia con respecto a la vulnerabilidad de ser víctima de violencia comunitaria; esto podría explicar la baja varianza de la variable de síntomas del TEPT explicada por la victimización indirecta. En conclusión, es necesario seguir realizando estudios que identifiquen factores de riesgo para el desarrollo de patologías en la salud mental, sobre todo al tener en cuenta la exposición a sucesos de victimización derivados de contextos con presencia de violencia comunitaria, ya que los resultados servirán para el desarrollo de programas de intervención, prevención y 
creación de políticas públicas que impacten en la calidad de vida de los habitantes de comunidades vulnerables.

\section{Referencias}

Almanza, A. M., Gómez, A. H., \& Gurrola, G. M. (2017). Victimización, resiliencia y salud mental de estudiantes de universidad en Tamaulipas, México. Revista Latinoamericana de Ciencias Sociales, Niñez y Juventud, 16(1), 345-360. doi: 10.11600/1692715x.16121

American Psychiatric Association. (2014). Manual Diagnostico $y$ Estadístico de los Trastornos Mentales (5. ${ }^{a}$ ed.). Arlington, VA: Editorial Médica Panamericana.

Baker, C. K., Norris, F. H., Diaz, D. M., Perilla, J. L., Murphy, A. D., \& Hill, E. G. (2005). Violence and PTSD in Mexico: gender and regional differences. Social Psychiatry and Psychiatric Epidemiology, 40(7), 519-28. doi: 10.1007/ s00127-005-0921-2

Bohnert, K. M., \& Breslau, N. (2011). Assessing the performance of the short screening scale for posttraumatic stress disorder in a large nationally-representative survey. International Journals of Methods in Psychiatric Research, 20(1), e1-e5. doi: 10.1002/mpr.331

Breslau, N., Peterson, E. L., Kessler, R. C., \& Schultz, L. R. (1999). Short screening scale for DSM-IV posttraumatic stress disorder. American Journal of Psychiatry, 156(6), 908-911. doi: 10.1176/ajp.156.6.908

Brown, J. R., Hill, H. M., \& Lambert, S. F. (2005). Traumatic stress symptoms in women exposed to community and partner violence. Journal of Interpersonal Violence, 20(11), 1478-1494. doi: 10.1177/ 0886260505278604

Buendía \& Laredo (2010). Los miedos de México. México, D. F: Buendía \& Laredo. [online] Recuperado de http://www. buendiaylaredo.com/publicaciones $/ 102 /$ media/losmiedos demexico.pdf

Caballero, M. A., \& Ramos, L. (2004). Violencia: una revisión del tema dentro del marco de trabajo de investigación en el Instituto Nacional de Psiquiatría. Salud Mental, 27(2), 21-30. Recuperado de http://inprf-cd.gob.mx/pdf/sm2702/ sm270221.pdf

Caballero, M. A., Ramos, L., \& Saltijeral, M. T. (2000). El trastorno por estrés postraumático y otras reacciones en las víctimas de robo a casa. Salud Mental, 23(1), 8-17. Recuperado de https://www.medigraphic.com/pdfs/salmen/ sam-2000/sam001b.pdf

Echeburúa, E. (2004). Superar un trauma. Madrid: Pirámide.

Elsaesser, C. (2018). The Longitudinal Relations between Dimensions of Community Violence Exposure and Developmental Outcomes among Adolescent Ethnic Minority males. Psychology of Violence, 8(4), 409-417. doi: 10.1037/vio0000140

Elsaesser, C., Gorman-Smith, D., Henry, D., \& Schoeny, M. (2017). The Longitudinal Relation between Community
Violence Exposure and Academic Engagement During Adolescence: Exploring Families' Protective Role. Journal of Interpersonal Violence, 32, 1-22. doi: $10.1177 / 0886260517708404$

Fowler P. J., Tompsett C. J., Braciszewski J. M., JacquesTiura A. J., \& Baltes B. B. (2009). Community violence: a meta-analysis on the effect of exposure and mental health outcomes of children and adolescents. Development \& Psychopathology, 21(1), 227-259. doi: 10.1017/ S0954579409000145

Instituto Nacional de Geografía, Estadística e Informática [INEGI]. (2018). Encuesta Nacional de Victimización y Percepción sobre Seguridad Pública (ENVIPE) 2018. Ciudad de México: INEGI [online]. Recuperado de https:// www.inegi.org.mx/programas/envipe/2018/

Javdani, S., Abdul-Adil, J., Suarez, L., Nichols, S. R., \& Farmer, A. D. (2014). Gender differences in the effects of community violence on mental health outcomes in a sample of low-income youth receiving psychiatric care. American Journal of Community Psychology, 53(3-4), 235-248. doi: 10.1007/s10464-014-9638-2

Leal, E. I., Vázquez, V. H., \& Cantú, O. N. (2013). Prevalencia de estrés postraumático en unidades de medicina familiar de seis ciudades de la frontera México-Estados Unidos de América. Atención Familiar, 20(4), 114-117. doi: 10.1016/ S1405-8871(16)30106-7

Medina-Mora, M., Borgues-Guimaraes, G., Lara, C., Ramos-Lira, L., Zambrano, J., \& Fleiz-Bautista, C. (2005). Prevalencia de sucesos violentos y de trastorno por estrés postraumático en la población mexicana. Salud Pública de México, 47(1), 8-22. doi: 10.1590/ S0036-36342005000100004

Monárrez, J. E., \& García, J. (2008). Violencia e inseguridad en la frontera norte de México. Nóesis. Revista de Ciencias Sociales y Humanidades, 17(34), 42-65. Recuperado de https://www.redalyc.org/pdf/859/85913301003.pdf

Montero, I., \& León, O. G. (2007). A guide for naming research studies in Psychology. International Journal of Clinical and Health Psychology, 7(3), 847-862. Recuperado de https:// www.redalyc.org/articulo.oa?id $=33770318$

Muratori, M., \& Zubieta, E. (2013). Miedo al delito y victimización como factores influyentes en la percepción del contexto social y clima emocional. Boletín de Psicología, 109, 7-18. Recuperado de https://www.uv.es/seoane/boletin/pre vios/N109-1.pdf

Organización Mundial de la Salud [OMS]. (2014). Informe sobre la situación mundial de prevención de la violencia. Ginebra, Suiza: OMS. Recuperado de https://apps.who. int/iris/bitstream/handle/10665/145089/WHO_NMH_ NVI_14.2_spa.pdf;jsessionid $=807174376958$ D63D 744700 FA4ËB66C 82 ?sequence $=1$

Organización Panamericana de la Salud [OPS]. (2002). Informe mundial sobre la violencia y la salud: resumen. Washington, D. C.: OPS. 
Orozco, R., Borges, G., Benjet, C., Medina-Mora, M. E., \& López-Carrillo, L. (2008). Traumatic life events and posttraumatic stress disorder among Mexican adolescents: results from a survey. Salud Publica de México, 50(supl. 1), S29-S37. doi: 10.1590/s0036-36342008000700006

Ramos, L., Caballero, M. A., \& Saltijeral, M. T. (1995). Efectos psicológicos y psicosociales en víctimas de delitos: un estudio cualitativo. Revista Psicología Social Personalidad, XI(2), 115-128. Recuperado de http://repositorio.inprf.gob. $\mathrm{mx} /$ handle/123456789/6775

Ramos, L., Esteban, R., Saltijeral, M. T., \& Caballero, M. A. (1997). Necesidades de atención a la salud mental en mujeres violadas. Salud Mental, 20(supl.), 47-54. Recuperado de http://www.inprf-cd.gob.mx/pdf/sm20s02/sm20s247.pdf

Ramos, L., Saltijeral, M. T., \& Caballero, M. A. (1995). Violencia delictiva: impacto en la salud mental. Psicología y Salud, 5, 153-166. Recuperado de http://repositorio.inprf. gob.mx/handle/123456789/6777

Rizo, L. E., Guevara, M. A., Hernández, M., \& SánchezSosa, J. J. (2018). A preliminary study of the prevalence of post-traumatic stress disorder, depression and anxiety symptoms in female adolescent's maltreatment victims in Mexico. Salud Mental, 41(3), 139-144. doi: 10.17711/ SM.0185-3325.2018.018

Rojas-Flores, L., Currier, J., Lin, E., Kulzer, R., \& Foy, D. (2013) "We are raising our children in fear"; War, community violence, and parenting practices in El Salvador. International Perspectives in Psychology: Research, practice, Consulation, 2(4), 269-285. doi: 10.1037/ipp0000009

Ruiz, J. I. (2007a). Cultura ciudadana, miedo al crimen y victimización: un análisis de sus interrelaciones desde la perspectiva del tejido social. Acta Colombiana, 10(1), 65-74. Recuperado de https://www.redalyc.org/pdf/798/79810107. pdf

Ruiz, J. I. (2007b). Procesos sociales relacionados con el miedo al crimen, la satisfacción con la policía y la victimización: el caso de la cultura ciudadana. International E-Journal of Criminal Sciences, 1(1), 1-24. Recuperado de https:/www. ehu.eus/ojs/index.php/inecs/article/view/17/19
Ruiz, J. I., \& Turcios, L. A. (2009). Percepción de seguridad, victimización y cultura ciudadana: sus relaciones en cinco contextos iberoamericanos. Pensamiento Psicológico, 6(13), 193-202. Recuperado de https://revistas.javeria nacali.edu.co/index.php/pensamientopsicologico/article/ view/125

Scarpa, A., Haden, S. C., \& Hurley, J. (2006). Community violence and symptoms of posttraumatic stress disorder: The moderating effects of coping and social support. Journal of Interpersonal Violence, 21(4), 446-469. doi: $10.1177 / 0886260505285726$

Sociedad Mexicana de Psicología. (2010). Código Ético del Psicólogo. México, D. F.: Trillas.

Vilalta, C. (2012). Los determinantes de la percepción de inseguridad frente al delito en México. (IDB working paper series, 381). México, D. F.: Banco Interamericano de Desarrollo. Recuperado de https://publications.iadb.org/ publications/spanish/document/Los-determinantes-de-lapercepci $\% \mathrm{C} 3 \% \mathrm{~B} 3 \mathrm{n}$-de-inseguridad-frente-al-delito-en$\mathrm{M} \% \mathrm{C} 3 \%$ A9xico.pdf

Walling, S. M., Eriksson, C. B., Putman, K. M., \& Foy, D. W. (2011). Community violence exposure, adverse childhood experiences, and posttraumatic distress among urban development workers. Psychological Trauma: Theory, Research, Practice, and Policy, 3(1), 42-49. doi: 10.1037/a0020566

Wright, E. M., Fagan, A. A., \& Pinchevsky, G. M. (2013). The effects of exposure to violence and victimization across life domains on adolescent substance use. Child Abuse Neglect, 37(11), 899-909. doi: 10.1016/j.chiabu.2013.04.010

Zimmerman, G. M., \& Posick, C. (2016). Risk Factors for and Behavioral Consequences of Direct Versus Indirect Exposure to Violence. American Journal of Public Health, 106(1), 178-188. doi: 10.2105/AJPH.2015.302920 